\title{
Neandertal DNA Sequences and the Origin of Modem Humans
}

\author{
Matthias Krings,* Anne Stone, ${ }^{\dagger}$ Ralf W. Schmitz, ${ }^{\ddagger}$ \\ Heike Krainitzki, § Mark Stoneking, ${ }^{\dagger}$ and Svante Pääbo* \\ *Zoological Institute \\ University of Munich \\ PO Box 202136 \\ D-80021 Munich \\ Germany \\ † Department of Anthropology \\ Pennsylvania State University \\ State College, Pennsylvania 16802 \\ $\ddagger$ Rheinisches Amt für Bodendenkmalpflege \\ Endenicher Strasse 133 \\ D-53115 Bonn \\ Germany \\ §Höhere Berufsfachschule für \\ präparationstechnische Assistenten \\ Markstrasse 185 \\ D-44799 Bochum \\ Germany
}

Summary

DNA was extracted from the Neandertal-type specimen found in 1856 in western Germany. By sequencing clones from short overlapping PCR products, a hitherto unknown mitochondrial (mt) DNA sequence was determined. Multiple controls indicate that this sequence is endogenous to the fossil. Sequence comparisons with human mtDNA sequences, as well as phylogenetic analyses, show that the Neandertal sequence falls outside the variation of modern humans. Furthermore, the age of the common ancestor of the Neandertal and modern human mtDNAs is estimated to be four times greater than that of the common ancestor of human mtDNAs. This suggests that Neandertals went extinct without contributing mtDNA to modern humans.

\section{Introduction}

Neandertals are a group of extinct hominids that inhabited Europe and western Asia from about 300,000 to 30,000 years ago. During part of this time they coexisted with modern humans. Based on morphological comparisons, it has been variously claimed that Neandertals: (1) were the direct ancestors of modern Europeans; (2) contributed some genes to modern humans; or (3) were completely replaced by modern humans without contributing any genes (reviewed in Stringer and Gamble, 1993; Trinkaus and Shipman 1993; Bräuer and Stringer, 1997). Analyses of molecular genetic variation in the mitochondrial and nuclear genomes of contemporary human populations have generally supported the third view, i.e., that Neandertals were a separate species that went extinct without contributing genes to modern humans (Cann et al., 1987; Vigilant et al., 1991; Hammer, 1995; Armour etal., 1996; Tishkoff et al., 1996). However, these analyses rely on assumptions, such as the absence of selection and a clock-like rate of molecular evolution in the DNA sequences under study, whose validity has been questioned (Wolpoff, 1989; Templeton, 1992). An additional and more direct way to address the question of the relationship between modern humans and Neandertals would be to analyze DNA sequences from the remains of Neandertals.

The reproducible retrieval of ancient DNA sequences became possible with the invention of the polymerase chain reaction (Mullis and Faloona, 1987; Pääbo et al., 1989). However, theoretical considerations, (Pääbo and Wilson, 1991; Lindahl 1993a) as well as empirical studies (Pääbo, 1989; Höss et al., 1996a), show that DNA in fossil remains is highly affected by hydrolytic as well as oxidative damage. Therefore, the retrieval of DNA sequences older than about 100,000 years is expected to be difficult, if not impossible, to achieve (Pääbo and Wilson, 1991). Fortunately, Neandertal remains fall within the age range that in principle allows DNA sequences to survive. It is noteworthy, though, that even among remains that are younger than 100,000 years most fail to yield amplifiable DNA sequences (Höss et al., 1996b). In addition, contamination of ancient specimens and extracts with modern DNA poses a serious problem (Handt et al., 1994a) that requires numerous precautions and controls. This is particularly the case when human remains are studied, since human DNA is the most common source of contamination. Therefore, a number of criteria need to be fulfilled before a DNA sequence determined from extracts of an ancient specimen can be taken to be genuine (Pääbo et al., 1989; Lindahl, 1993b; Handt et al., 1994a; Handt et al., 1996).

Since 1991, the Neandertal-type specimen, found in 1856 near Düsseldorf, Germany, has been the subject of an interdisciplinary project of the Rheinisches Landesmuseum Bonn, initiated and led by R. W. S. (Schmitz et al., 1995; Schmitz, 1996). As a part of this project, a sample was removed from the Neandertal specimen for DNA analysis. Here, we present the sequence of a hypervariable part of the mtDNA control region derived from this sample. We describe the evidence in support of its authenticity and analyze the relationship of this sequence to the contemporary human mtDNA gene pool.

\section{Results}

\section{Amino Acid Racemization}

A $3.5 \mathrm{~g}$ section of the right humerus was removed from the Neandertal fossil (Figure 1). It has previously been shown that ancient specimens exhibiting high levels of amino acid racemization do not contain sufficient DNA for analysis (Poinar et al., 1996). To investigate whether the state of preservation of the fossil is compatible with DNA retrieval, we therefore analyzed the extent of amino acid racemization. Samples of $10 \mathrm{mg}$ were removed from the periostal surface of the bone, from the compact 


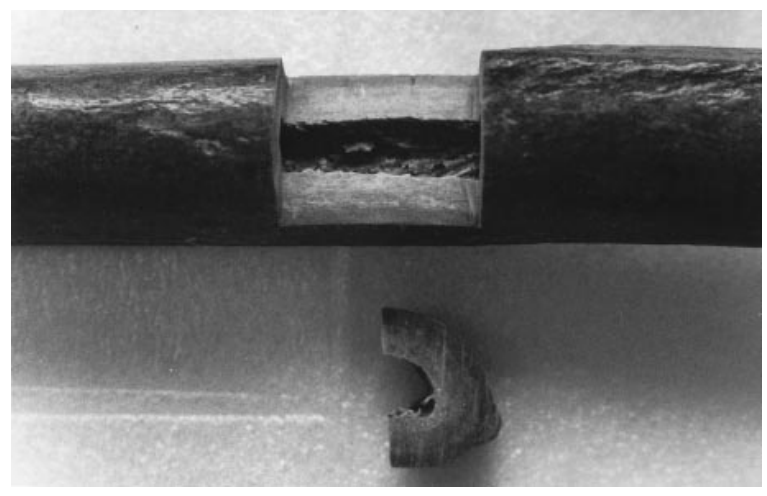

Figure 1. Sample Removed from the Right Humerus of the Neandertal-Type Specimen

cortical bone, and from the endostal surface of the marrow cavity. Samples were also removed from remnants of a varnish, with which the specimen has been treated at least twice. The samples were hydrolyzed under acid conditions, and the released amino acids were analyzed using high performance liquid chromatography and fluorescent detection (Poinar et al., 1996). Table 1 shows that the total amounts of the amino acids detected in the Neandertal bone are $20 \%-73 \%$ of those in modern bone and more than two orders of magnitude higher than in the varnish, indicating that the results do not reflect the amino acid content of the varnish. Furthermore, the absolute and relative amounts of the amino acids analyzed (e.g., the ratio of glycine to aspartic acid) are similar in the three Neandertal samples and comparable to those of a contemporary bone. Most importantly, the ratio of the $D$ to the $L$ enantiomers of aspartic acid in the three Neandertal samples varies between 0.11 and 0.12 , which is in the range compatible with DNA survival (Poinar et al., 1996). Thus, the extent of amino acid racemization in the Neandertal fossil suggests that it may contain amplifiable DNA.

\section{DNA Extraction and Amplification}

DNA was extracted from $0.4 \mathrm{~g}$ of the cortical compact bone. Previous experience shows that ancient DNA tends to be degraded and damaged to an extent that makes amplification of segments of mtDNA longer than 100-200 bp difficult (Pääbo, 1989). Therefore, two primers $(L 16,209, H 16,271)$ that amplify a 105-bp-segment of the human mtDNA control region (including primers) were used to perform amplifications from the bone extract as well as from an extraction control. An amplification product was obtained in the bone extract but not in the control (data not shown). In a subsequent experiment, this was repeated and the same results were obtained.

\section{Sequence Variation of the Amplification Product}

The two amplification products were cloned in a plasmid vector and 18 and 12 clones, respectively, were sequenced (Figure 2, extract A). Twenty-two of the 30 clones contained seven nucleotide substitutions and one insertion of an adenine residue, when compared to the standard human reference sequence (Anderson et al., 1981). Three of these eight differences to the reference sequence were individually lacking in a total of five of the clones. In addition, among the 27 clones were nine differences that each occurred in one clone, three differences that occurred in two clones and one that occurred in three clones, respectively. Such changes that are present in only a few clones are likely to be due to misincorporations by the DNA polymerase during $P C R$, possibly compounded by damage in the template DNA. In addition, some of these could be due to mitochondrial heteroplasmy, which may be more common in humans than often assumed (Comas et al., 1995; Ivanov et al., 1996) and is abundant in some mammalian species (Petri et al., 1996). Of the remaining three clones, two were identical to the reference sequence, and the third clone differed from the reference sequence at one position.

Thus, the amplification product was composed of two classes of sequences, a minor class represented by three clones that is similar to the human reference sequence, and another class represented by 27 clones that exhibits substantial differences from it. The former class of molecules probably reflects contamination of

Table 1. Racemization Results for Three Neandertal Bone Samples, Varnish from the Neandertal Fossil, and Modern Bone

\begin{tabular}{|c|c|c|c|c|c|}
\hline & Periostal Surface & Compact Bone & Endostal Surface & Varnish & Modern Bone \\
\hline Total (ppm) & 23,167 & 83,135 & 53,888 & 145 & 113,931 \\
\hline Aspartic acid (\%) & 7.8 & 8.3 & 7.4 & 10 & 8.3 \\
\hline Serine $(\%)$ & 0.7 & 0.7 & 0.7 & 2 & 0.6 \\
\hline Glutamic acid (\%) & 20.2 & 20.1 & 20.2 & 22 & 19.9 \\
\hline Glycine (\%) & 49.5 & 49.0 & 50.2 & 22 & 51.8 \\
\hline Alanine (\%) & 14.4 & 14.0 & 14.0 & 11 & 11.1 \\
\hline Valine $(\%)$ & 3.5 & 3.9 & 3.9 & 23 & 3.9 \\
\hline Isoleucine (\%) & 0.5 & 0.5 & 0.6 & 1 & 0.7 \\
\hline Leucine (\%) & 3.4 & 3.3 & 3.2 & 9 & 3.6 \\
\hline Glycine/aspartic acid & 6.3 & 5.9 & 6.8 & 2.1 & 6.2 \\
\hline D/L aspartic acid & 0.117 & 0.114 & 0.110 & ND & 0.05 \\
\hline $\mathrm{D} / \mathrm{L}$ alanine & 0.006 & 0.007 & 0.004 & 0.08 & 0.01 \\
\hline$D / L$ leucine & 0.005 & ND & ND & ND & ND \\
\hline
\end{tabular}

Comparison of the amino acid analysis of the Neandertal bone, varnish removed from the bone surface, and a two-year-old bone sample. Given are the total amounts of the amino acids analyzed (ppm, parts per million), the amino acid compositions in percentages of the eight amino acids analyzed, and the D/L-ratios for three amino acids. ND, no detectable D form. 


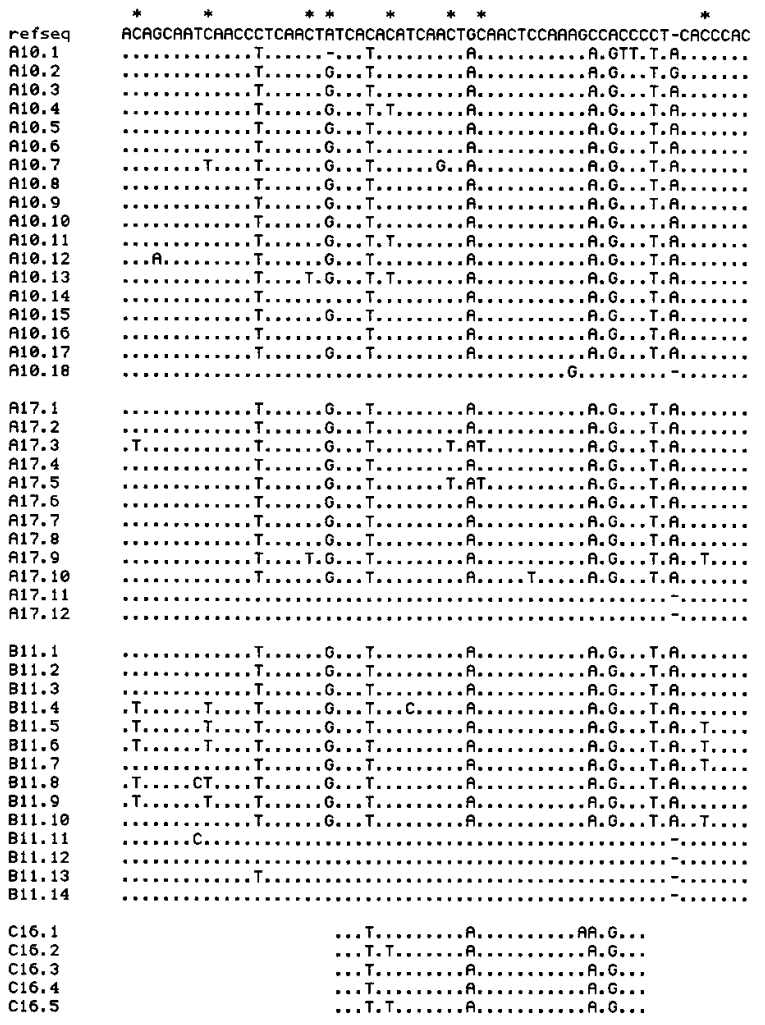

Figure 2. The DNA Sequences of Clones Derived from Four Amplifications of the Mitochondrial Control Region from the Neandertal Fossil

Dots indicate identity to a human reference sequence (Anderson et al., 1981) given above. The clone designations consist of a letter ( $A$ $B, C$ ) indicating the DNA extract followed by a number indicating the amplification reaction, as well as a number after the period identifying the particular clone. Extracts $\mathrm{A}$ and $\mathrm{B}$ were performed at the University of Munich; extract $C$, at Penn State University. Clones derived from different amplifications are separated by a blank line. Asterisks identify sequence positions where more than one clone differs from the majority of sequences. For the three upper amplifications (performed at the University of Munich) primers L16,209 (5'-CCC CAT GCT TAC AAG CAA GT-3') and H16,271 (5'GTG GGT AGG TTT GTT GGT ATC CTA-3') were used. For the bottom amplification (performed at Penn State University) the primers NL16,230 (5'-GCA CAG CAA TCA ACC TTC AAC TG-3') and NH16,262 (5'-GTA GAT TTG TTG ATA TCC TAG TGG GTG TAA-3') were used.

the specimen, which is likely to have occurred during handling and treatment of the specimen during the 140 years since its discovery. The other class of sequences is not obviously of modern origin. Further experiments were therefore performed to determine if this class is endogenous to the Neandertal fossil.

\section{Quantitation of Putative Neandertal DNA}

Amplifications that start from more than 1000 ancient template molecules tend to yield reproducible results, while amplifications starting from fewer molecules tend to yield results that vary between experiments, due to misincorporations during the early cycles of the PCR as well as due to sporadic contamination (Handt et al.,

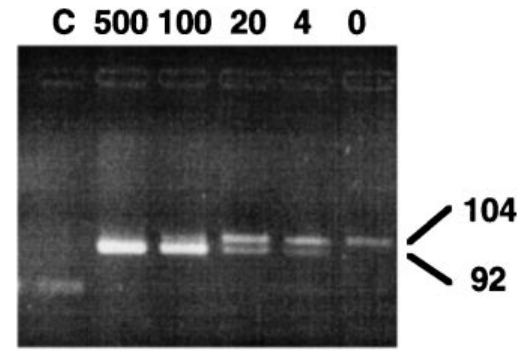

Figure 3. Quantitation of the Putative Neandertal mtDNA

A dilution series of a competitor construct carrying the putative Neandertal sequence with a $12 \mathrm{bp}$ deletion was added to $2.5 \mu \mathrm{l}$ of extract $A$ from the fossil. P rimers used were specific for the putative Neandertal sequence. Above the lanes, the approximate numbers of cd LWcompetitor molecules added are indicated. The control amplification (C) contained neither competitor nor Neandertal extract.

1996). Therefore, the number of template molecules representing the putative Neandertal sequence in the extract was determined by quantitative PCR. To this end, a molecule representing the putative Neandertal sequence but carrying a 12 bp deletion was constructed. To each step in a dilution series of this construct, a constant amount of extract was added and amplifications were performed using primers that are specific for a $104 \mathrm{bp}$ product of the putative Neandertal sequence and that do not amplify contemporary human sequences. The results (Figure 3 ) show that on the order of 10 putative Neandertal molecules exist per microliter of extract and thus that amplifications starting from 5 $\mu$ l of extract are initiated from approximately 50 template molecules. However, due to variation in the efficiency of individual primer pairs, and stochastic variation in the number of template molecules added to an individual amplification, some amplifications may start from fewer (or even single) molecules. This makes nucleotide misincorporations in early cycles of the amplific ation reaction likely to affect a large proportion of the molecules in the final amplification product. Such misincorporations may be frequent since the template molecules are likely to carry miscoding base modifications (Höss et al., 1996a). To detect this type of sequence change, amplifications were performed such that each sequence position to be determined was covered by at least two independent $P C R$ reactions. The products of each PCR reaction were independently cloned and the sequences determined from multiple clones.

\section{Authenticity of Sequences}

The inadvertent amplification of small amounts of contemporary DNA is a major source of erroneous results in the study of ancient DNA sequences (Pääbo et al., 1989; Lindahl, 1993b; Handt et al., 1994a). Such contamination may result in the amplific ation of not only contemporary organellar mtDNA but also of nuclear insertions of mtDNA (Collura and Stewart, 1995; van der Kuyl et al., 1995; Zischler et al., 1995). Several experiments were performed in order to exclude modern DNA, including a nuclear insertion of mtDNA, as the source of the putative Neandertal sequence. 
Since nuclear insertions are less numerous than mitochondrial genomes in the organelles, any single insertion sequence is expected to represent a major proportion of an amplification product only in cases where a primer favors the amplification of an insertion sequence over the corresponding mtDNA sequence. This occurs when mismatches to the primer in the mtDNA make the priming of an insertion more efficient than that of the organellar mtDNA (Handt et al., 1996). Therefore, the preferential amplification of an insertion sequence is expected to be restricted to a particular primer. In order to elucidate whether the putative Neandertal sequence is seen only when a particular primer is used, primers were exchanged such that first the $5^{\prime}$ primer was replaced by a primer located outside the previous amplification product $(L 16,122)$, and 13 clones of this amplification product were sequenced (Figure 4, clones A7.1-13). All 13 clones showed the same eight differences from the reference sequence that were previously observed, as well as nine differences in the region that was not included in the earlier amplification. In addition, one difference was observed in one clone, as well as length variation in a homopolymer of cytosine residues, previously described to be of variable length in humans (Bendall and Sykes, 1995).

The 3 -primer from the first amplification was then replaced by a primer $(\mathrm{H} 16,379)$ located outside the initial amplification product, and 13 clones of this amplification product were sequenced (Figure 5, clones A12.1-13). All 13 clones contained the same eight differences in the region overlapping the previous amplifications, as well as seven differences in the region not covered in the previous amplifications. In addition, two substitutions and one deletion occurred in one clone, and one other substitution occurred in a different clone. Furthermore, in a subsequent amplification from another extract where both primers (L16,254-H16,379) differed from the initial amplification, all four differences located in the segment included in the first amplifications were observed in all 8 clones sequenced (Figure 5, clones B13.1-8). Thus, the retrieval of the putative Neandertal sequence is not dependent on the primers used. Furthermore, most primer combinations yield a large excess of clones representing the putative Neandertal sequence over clones similar to contemporary human mtDNA.

To further exclude the possibility that the sequence may represent a nuclear insertion, primers for the putative Neandertal sequence were constructed that do not amplify human mtDNA. In control experiments where various amounts of a cloned copy of the putative Neandertal sequence were mixed with human DNA, these primers were able to detect about 20 copies of the cloned sequence in $50 \mathrm{ng}$ of total human DNA, i.e., less than one copy per genome equivalent (data not shown). When these primers were used to amplify DNA isolated from 15 Africans, 6 Europeans, and 2 Asians, no amplification products were obtained (data not shown), indicating that this sequence is not present in the genome of modern humans.

To test whether the extraction and amplification of the putative Neandertal sequence is reproducible, an additional independent DNA extraction was performed from $0.4 \mathrm{~g}$ of the bone. When the primers L16,209 and $\mathrm{H} 16,271$ were used in an amplification from this extract and the product cloned (Figure 2, extract $B$ ), ten clones carried the eight differences from the reference sequence observed in the amplifications from the first extract, as well as two changes affecting single clones. In addition, three sequence positions carried changes occurring in five and four clones. These changes were not observed in combination in the previous four amplifications covering this sequence segment. Since they occurred in only one amplification product, they are probably due to polymerase errors in the early cycles of the PCR, possibly compounded by in vitro recombination induced by damage and degradation of template DNA molecules (Pääbo et al., 1990). Four clones were similar to the human reference sequence. Thus, although the amplification products clearly derive from few template molecules, the putative Neandertal sequence is present in a DNA extract independently prepared from the fossil.

To further investigate whether the results are due to laboratory-specific artifacts or contamination, an additional bone sample of $0.4 \mathrm{~g}$ was sent to the Anthropological Genetics Laboratory at Pennsylvania State University where a DNA extraction was performed. When the primers (L16,209 and $\mathrm{H} 16,271$ ), which had previously resulted in a product that contained both the putative Neandertal sequence and contemporary human mtDNA sequences (Figure 2 ) were used in amplifications from this extract, 15 of the resulting clones yielded a DNA sequence that was identical to the experimenter (A. S.), while two yielded sequences that differed by one and two substitutions from the reference sequence, respectively. However, when primers specific for the putative Neandertal sequence (NL16,230 and $\mathrm{NH16,262)}$ were used, 5 out of 5 clones yielded the putative Neandertal sequence (Figure 2, extract $C$ ). Thus, while this third independent extract contains a larger amount of contemporary human DNA, probably stemming from laboratory contamination, it confirms that the putative Neandertal sequence is present in the fossil specimen.

In summary, these experiments indic ate that the putative Neandertal sequence does not originate from a nuclear mtDNA insertion and that it is endogenous to the fossil. It furthermore falls outside the variation of the mtDNA gene pool of modern humans (see below). We therefore conclude that it is derived from the mitochondrial genome of the Neandertal individual.

\section{Determination of the Neandertal mtDNA Sequence}

The entire sequence of hypervariable region I of the mtDNA control region (positions 16,023 to 16,400 ; Anderson et al., 1981) was determined. Since the state of preservation of the DNA allowed only short fragments to be amplified, this was achieved by several overlapping amplifications. Furthermore, since the quantitation experiments indicated that some amplifications might start from single molecules, and thus that misincorporations in early cycles of the amplification might be misinterpreted as sequence differences (Handt et al., 1996), all sequence positions were determined from at least two independent amplifications. At five sequence positions, two amplifications yielded discordant results, i.e., all 

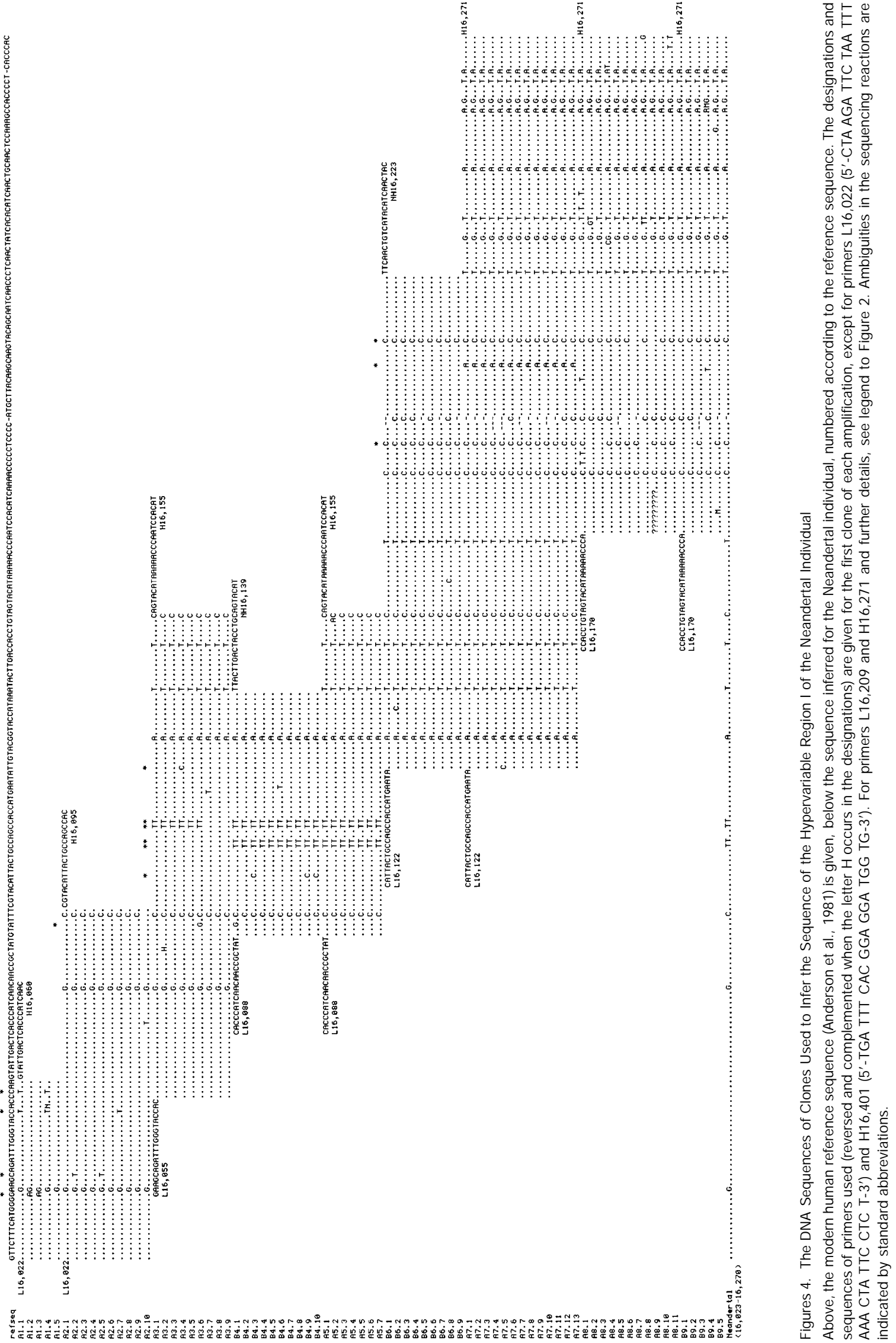


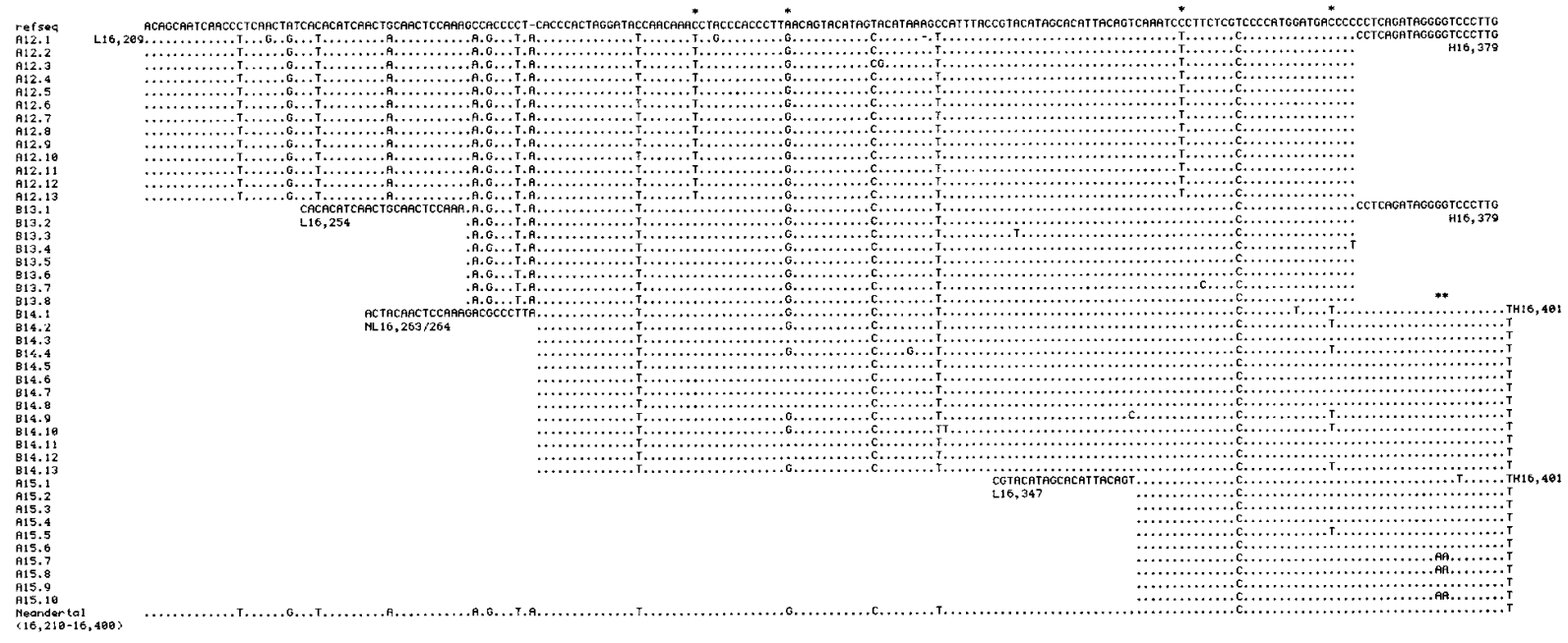

Figure 5. The DNA Sequences of Clones Used to Infer the Sequence of the Hypervariable Region I of the Neandertal Individual Above, the modern human reference sequence (Anderson et al., 1981) is given, below the sequence inferred for the Neandertal individual, numbered according to the reference sequence. The designations and sequences of primers used (reversed and complemented when the letter $\mathrm{H}$ occurs in the designations) are given for the first clone of each amplification, except for primers L16,022 (5'-CTA AGA TTC TAA TTT AAA CTA TTC CTC T-3') and H16,401 (5'-TGA TTT CAC GGA GGA TGG TG-3'). For primers L16,209 and H16,271 and further details, see legend to Figure 2 . Ambiguities in the sequencing reactions are indicated by standard abbreviations.

clones in one amplification differed at a position from all clones in another amplification. For these positions, clones from at least one more independent amplification were sequenced. In all cases, all clones from the subsequent amplification products carried one of the two bases at the five positions in question. At 23 positions, differences were found between two or more clones, either within one amplification or in different amplifications. In those cases, the sequence present in the majority of clones was scored. Figures 4 and 5 show how 123 clones from 13 amplifications were used to determine $379 \mathrm{bp}$ from the Neandertal individual.

\section{Sequence Comparisons}

When the Neandertal DNA sequence is compared to the human reference sequence, 27 differences are seen outside the heteroplasmic cytosine homopolymer (Bendall and Sykes, 1995) (Figure 4). Of these 27 differences, 24 are transitions, two are transversions, and one represents an insertion of a single adenosine residue.

The Neandertal sequence was compared to a collection of 2051 human and 59 common chimpanzee sequences over $360 \mathrm{bp}$ of the sequence determined from the Neandertal (positions 16,024 to 16,383). Among the 27 nucleotide differences to the reference sequence found in this segment, 25 fall among the 225 positions that vary in at least one of the human sequences, and one of the two remaining positions varies among the chimpanzees. Thus, the types of differences observed (e.g., an excess of transitions over transversions), and the positions in the Neandertal sequence where they occur, reflect the evolutionary pattern typical of mtDNA sequences of extant humans and chimpanzees.

The Neandertal sequence was compared to 994 contemporary human mitochondrial lineages, i.e., distinct sequences occurring in one or more individuals, found in 478 Africans, 510 Europeans, 494 Asians, 167 Native Americans and 20 individuals from Australia and Oceania (S. Meyer, personal communication). Whereas these modern human sequences differ among themselves by an average of $8.0 \pm 3.1$ (range 1-24) substitutions, the difference between the humans and the Neandertal sequence is $27.2 \pm 2.2$ (range 22-36) substitutions. Thus, the largest difference observed between any two human sequences was two substitutions larger than the smallest difference between a human and the Neandertal. In total, $0.002 \%$ of the pairwise comparisons between human mtDNA sequences were larger than the smallest difference between the Neandertal and a human.

The Neandertal sequence, when compared to the mitochondrial lineages from different continents, differs by $28.2 \pm 1.9$ substitutions from the European lineages, $27.1 \pm 2.2$ substitutions from the African lineages, $27.7 \pm$ 2.1 substitutions from the Asian lineages, $27.4 \pm 1.8$ substitutions from the American lineages and $28.3 \pm$ 3.7 substitutions from the Australian/Oceanic lineages. Thus, whereas the Neandertals inhabited the same geographic region as contemporary Europeans, the observed differences between the Neandertal sequence and modern Europeans do not indicate that it is more closely related to modern Europeans than to any other population of contemporary humans.

When the comparison was extended to 16 common chimpanzee lineages (Figure 6), the number of positions in common among the human and chimpanzee sequences was reduced to 333 (Morin et al., 1994). This reduced the number of human lineages to 986 . The average number of differences among humans is $8.0 \pm 3.0$ (range 1-24), that between humans and the Neandertal, $25.6 \pm 2.2$ (range 20-34), and that between humans 
and chimpanzees, $55.0 \pm 3.0$ (range 46-67). Thus, the average number of mtDNA sequence differences between modern humans and the Neandertal is about three times that among humans, but about half of that between modern humans and modern chimpanzees.

An insertion of a portion of the mitochondrial control region on chromosome 11 has been inferred to represent an outgroup to the modern human mtDNA gene pool (Zischler et al., 1995). When its sequence was compared to the human and Neandertal sequences, 294 positions could be compared and the number of human lineages was reduced to 970 . In this case, the differences between humans and the Neandertal sequence are $25.5 \pm 2.1$ (range 20-34) whereas between humans and the insertion sequence there are $21.3 \pm 1.7$ (range 16-27) differences. Thus, although the distributions of the distances overlap, they suggest that the insertion of a portion of the mtDNA control region to chromosome 11 may have occurred after the divergence of the Neandertal and modern human mtDNA gene pools. This is compatible with the notion that the Neandertal sequence diverged from the lineage leading to the current human mtDNA gene pool well before the time of the most recent common ancestor of human mtDNAs.

\section{Phylogenetic Analyses}

To further investigate the relationship of the Neandertal mtDNA sequence to contemporary human mtDNA variation, phylogenetic tree reconstructions were performed. A neighbor-joining tree (Saitou and Nei, 1987) of the 16 chimpanzee lineages, the Neandertal sequence, and the 986 human lineages was constructed (Figure 7a). This tree shows the Neandertal sequence diverging prior to the divergence of the human mtDNA lineages. To estimate the support for this relationship, a likelihood mapping statistic (Strimmer and von Haeseler, 1997) was used. In this analysis, all possible quartets involving the Neandertal sequence, one of the chimpanzee lineages, and two representatives out of 100 human lineages (randomly selected from the 986 human lineages) were analyzed, and the likelihoods for the three possible groupings of the Neandertal, chimpanzee, and human

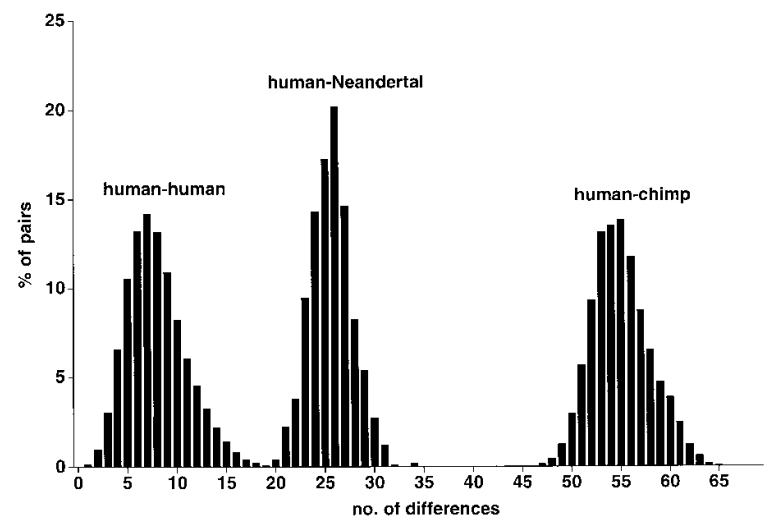

Figure 6. Distributions of Pairwise Sequence Differences among Humans, the Neandertal, and Chimpanzees

$X$ axis, the number of sequence differences; $Y$ axis, the percent of pairwise comparisons. sequences were plotted for each quartet. An example of this analysis is shown in Figure $7 \mathrm{~b}$. A different random subset of 100 human lineages was then chosen and the analysis repeated; in 40 such analyses, an average of $89 \%$ (range $84 \%-93 \%$ ) of the quartets grouped the two human sequences together. Thus, the phylogenetic analyses agree with the pairwise comparisons of sequence differences in placing the Neandertal mtDNA sequence outside the variation of modem human mtDNA.

\section{Age of the Neandertal/Modern Human mtDNA Ancestor}

To estimate the time when the most recent ancestral sequence common to the Neandertal and modern human mtDNA sequences existed, we used an estimated divergence date between humans and chimpanzees of 4-5 million years ago (Takahata et al., 1995) and corrected the observed sequence differences for multiple substitutions at the same nucleotide site (Tamura and Nei, 1993). This yielded a date of 550,000 to 690,000 years before present for the divergence of the Neandertal mtDNA and contemporary human mtDNAs. When the age of the modern human mtDNA ancestor is estimated using the same procedure, a date of 120,000 to 150,000 years is obtained, in agreement with previous estimates (Cann et al., 1987; Vigilant et al., 1991). Although these dates rely on the calibration point of the chimpanzeehuman divergence and have errors of unknown magnitude associated with them, they indicate that the age of the common ancestor of the Neandertal sequence and modern human sequences is about four times greater than that of the common ancestor of modern human mtDNAs.

\section{Rooting of Modern Human mtDNA Gene Pool}

The phylogenetic tree (Figure 7a) shows the first three human branches to be composed of seven African mtDNA sequences, with the first non-African sequences appearing only in the fourth branch. This branching pattern would indicate that the ancestor of the mtDNA gene pool of contemporary humans lived in Africa (cf. von Haeseler et al., 1996). When the statistical support for these branches was assessed with the likelihood mapping approach using the Neandertal sequence as an outgroup, these three branches weresupported by $91 \%$, $91 \%$, and $92 \%$ of quartets, respectively. When the seven African sequences in the first three branches were tested together with the 684 non-African lineages in the database and the Neandertal sequence, they grouped with the latter in $97 \%$ of all quartets analyzed. Thus, overall, the results suggest an African origin of the human mtDNA gene pool, as has been claimed when chimpanzee sequences (Vigilant et al., 1991; Hedges et al., 1992) and a nuclear insertion of the mitochondrial control region (Zischler et al., 1995) were used in similar analyses.

\section{Discussion}

DNA Preservation in the Neandertal Fossil

Based on its "classical" morphology, the undated Neandertal fossil is thought to be between 30,000 and 100,000 
a

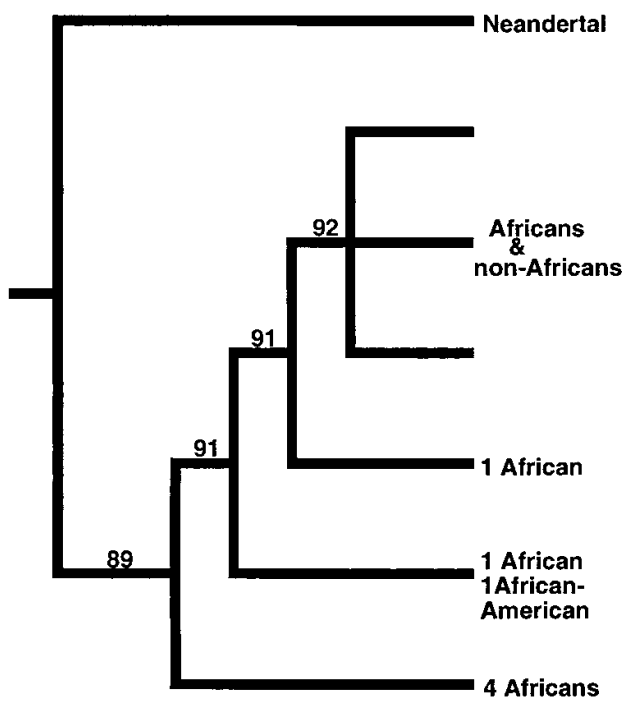

b
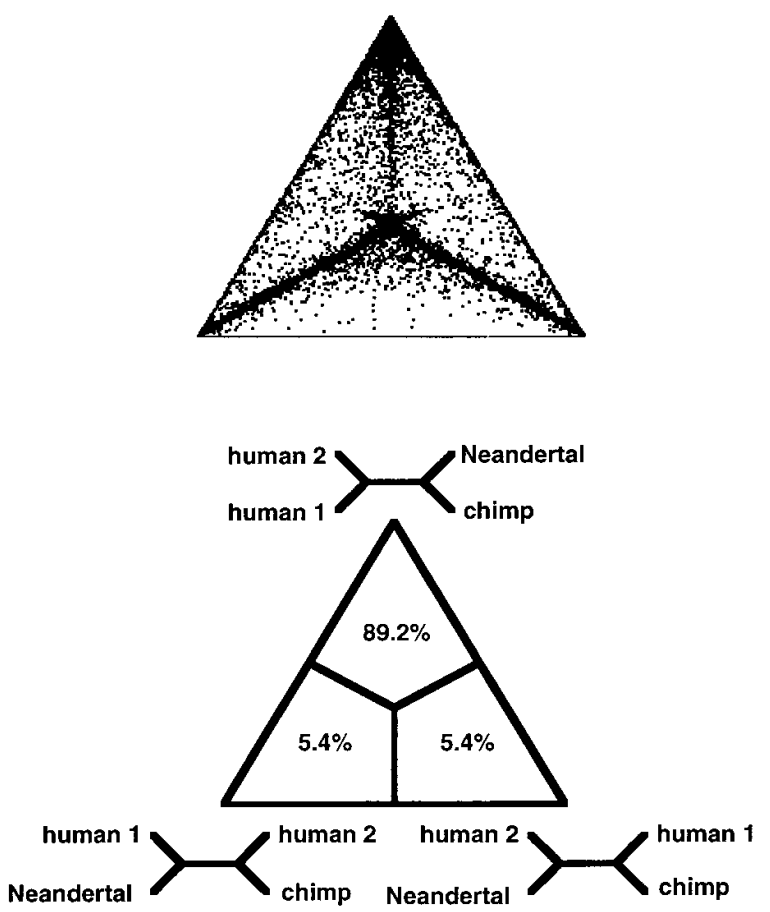

Figure 7. A Schematic Phylogenetic Tree Relating the Neandertal mtDNA Sequence to 986 Modern Human mtDNA Sequences and Likelihood Mapping Analysis Showing the Support for Various Groupings of Neandertal, Human, and Chimpanzee Sequences

(a) The tree was rooted with 16 chimpanzee mtDNA lineages. For clarity, only the first five branches without their internal branching structures but with their geographical states are shown. Numbers on internal branches refer to quartet puzzling probabilities. To calculate these, all possible combinations of the Neandertal sequence, one of 16 chimpanzee lineages, and two of 100 lineages chosen at random from among 986 human lineages were analyzed.

(b) For each such quartet of sequences, the likelihoods for each of the three possible phylogenetic arrangements are plotted in a triangle (b, upper panel) where the tips indicate absolute support for one of the arrangements (b, lower panel). The percentage of the quartets favoring the grouping of the Neandertal sequence with the chimpanzee to the exclusion of the two human lineages is found in the upper of the three areas. A total of 40 such analyses with different random sets of human mtDNA lineages were carried out and the average of these is given in the tree. The other internal branches were similarly analyzed.

years old (Stringer and Gamble, 1993). It is thus among the oldest specimens for which the chemical stability of DNA would seem to allow for the retrieval of endogenous DNA (Pääbo and Wilson, 1991; Lindahl, 1993a). Furthermore, the extent of amino acid racemization indicates that preservation conditions of the Neandertal fossil have been compatible with DNA preservation (Poinar et al., 1996). In agreement with this, the quantitation shows that an extract of $0.4 \mathrm{~g}$ of bone contains about 10001500 Neandertal mtDNA molecules of length $100 \mathrm{bp}$. Thus, mitochondrial DNA sequences can be retrieved from the fossil. However, this result also indicates that single-copy nuclear DNA sequences, which are a hundred- to a thousand-fold less abundant than mtDNA in most cells, would be impossible to reproducibly amplify from the extracts. This is reminiscent of the situation in most other archaeological remains (e.g., Handt et al., 1994b, 1996).

Several factors complicate the determination of mtDNA sequences from the Neandertal fossil. The low number of preserved mtDNA molecules poses problems since misincoporations during the initial cycles of the amplification will become represented in a large fraction of the molecules in the final amplification product. Such misincorporations may be particularly likely in the case of ancient DNA, which often contains lesions that can enhance misincorporations (Höss et al., 1996a). In addition, the Neandertal extracts contain sequences that are probably derived from contemporary humans. This is not unexpected since the specimen has been extensively handled during 140 years. A further complication is that exogenous as well as endogenous molecules, both sometimes carrying substitutions from early cycles of the amplification, may recombine with each other during the amplification process. Such "jumping PCR" is induced by strand breaks and DNA damage and may itself introduce sequence changes (Pääbo et al., 1990).

Fortunately, neither misincorporations nor jumping phenomena are expected to show a great specificity for certain sequence positions. Therefore, even when an amplification starts from a single molecule and an error occurs in the first cycle of the PCR such that it becomes represented in all resulting molecules, one will be alerted to the problem if two independent amplifications are analyzed since they will yield different sequences. A third amplification can then be analyzed to determine which of the two sequences is reproducible and hence authentic. Taken together, the results (Figures 4 and 5) show that misincorporations are a fairly frequent phenomenon in the Neandertal extracts, contributing to variation seen at 64 of the 378 sequence positions determined. Furthermore, some amplifications start from very 
few or single molecules since amplifications yielded completely discordant results at five positions. In contrast, jumping PCR, which is a frequent phenomenon in extracts of some ancient specimens (Handt et al., 1994b), does not seem to play a major role in the case of the Neandertal fossil, although some clones (Figure 2 , extract $B$ ) are likely to be the result of this process.

\section{Implications for Modern Human Origins}

Both pairwise sequence comparisons and phylogenetic analyses tend to place the Neandertal mtDNA sequence outside modern human mtDNA variation. Furthermore, the divergence between the Neandertal mtDNA sequence and the modern human mitochondrial gene pool is estimated to be about four-fold older than the diversity of the modern human mtDNA gene pool. This shows that the diversity among Neandertal mtDNA sequences would have to be at least four times larger than among modern humans in order for other Neandertal sequences to be ancestral to modern human sequences. Thus, although based on a single Neandertal sequence, the present results indic ate that Neandertals did not contribute mtDNA to modern humans.

These results do not rule out the possibility that Neandertals contributed other genes to modern humans. However, the view that Neandertals would have contributed little or nothing to the modern human gene pool is gaining support from studies of molecular genetic variation at nuclear loci in humans (Hammer, 1995; Armour et al., 1996; Tishkoff et al., 1996). It is also in agreement with assessments of the degree of morphological difference between Neandertal skeletal remains and modern humans (e.g., Rak, 1993; Zollikofer et al., 1995; Hublin et al., 1996; Schwartz and Tattersall, 1996) that would classify Neandertals and modern humans as separate species.

Given the placement of the Neandertal mtDNA sequence outside the range of modern human mtDNA variation, it can be used as an outgroup in phylogenetic analyses to assess the geographic origin of the human mtDNA ancestor. Initial claims that Africa was the most likely geographic source of contemporary human mtDNA variation (Cann et al., 1987; Vigilant et al., 1991) were challenged by subsequent reanalyses that found the original phylogenetic analyses to be inadequate ( $M$ addison, 1991; Templeton, 1992; Hedges et al., 1992). However, new methods of phylogenetic analysis have continued to support an African origin of human mtDNA variation (Penny et al., 1995), as has the use of a nuclear mtDNA insertion as an outgroup (Zischler et al., 1995). When the Neandertal mtDNA sequence is used to root a neighbor joining tree of modern human mtDNA sequences (Figure 7a), the first three branches consist exclusively of African sequences. The Neandertal mtDNA sequence thus supports a scenario in which modern humans arose recently in Africa as a distinct species and replaced Neandertals with little or no interbreeding.

\section{Implications for Neandertal Genetics}

It is interesting to compare the mtDNA date for the divergence between Neandertals and modern humans of 550,000 to 690,000 years ago with dates derived from other sources of information. For example, the fossil record indicates a likely minimum date for the divergence between modern humans and Neandertals of 250,000-300,000 years (Stringer, 1997), while the archaeological record also puts the divergence between modern humans and Neandertals at about 300,000 years (Foley and Lahr, 1997). A date of over 500,000 years for the molecular divergence between Neandertal and human mtDNAs is in excellent agreement with the palaeontological and archaeological record since the divergence of genes is expected to predate the divergence of populations by an amount that reflects the level of polymorphism in the ancestral species (Nei, 1987). Thus, if the palaeontological and archaeological estimates for the divergence of the Neandertal and human populations are accurate, and the mIDNA estimate for the molecular divergence is also accurate, this would indicate that the diversity of the mtDNA gene pool in the ancestral species (presumably Homo erectus) from which Neandertals and humans evolved, was at least as great as that of modern humans.

It must be emphasized that the above conclusions are based on a single individual sequence; the retrieval and analysis of mtDNA sequences from additional Neandertal specimens is obviously desirable. If this proves possible, then the potential exists to address several questions concerning Neandertals that hitherto could be studied exclusively by morphological and archaeological approaches. For example, the genetic relationship between Neandertal populations in Europe and in western Asia could be explored, as could the demographic history of Neandertal populations, using methods that have been applied to investigate the demographic history of modern human populations (Harpending et al., 1993; von Haeseler et al., 1996).

\section{A Cautionary Note}

Remains of animals found in association with Neandertal remains in other parts of Europe have failed to yield amplifiable DNA and/or display levels of amino acid racemization that make the prospect of retrieving DNA bleak (A. Cooper and H. Poinar, personal communication). It is therefore possible that the type specimen may be fairly unique in containing amplifiable endogenous DNA. Thus, we strongly recommend that valuable Neandertal specimens should not be subjected to destructive sampling before the analysis of associated animal fossils, and/or the application of some other method that requires minimal destruction of specimens (such as amino acid racemization), has yielded evidence that DNA may survive in the fossil.

\section{Experimental Procedures}

\section{Sampling}

Protective clothing was worn throughout the sampling procedure. Instruments used were treated with $1 \mathrm{M} \mathrm{HCl}$ followed by extensive rinsing in distilled water. After removal, the sample was immediately put into a sterile tube for transport to Munich. All subsequent manipulations of the sample, and experimental procedures prior to cycling of $P C R$ reactions, were carried out in laboratories solely dedicated to the analysis of archaeological specimens, where protective clothing, separate equipment and reagents, UV irradiation, and other measures to minimize contamination are used routinely.

\section{Amino Acid Analysis}

Samples of $10 \mathrm{mg}$ of bone powder and varnish were removed by drilling and were hydrolyzed in $1 \mathrm{ml} 6 \mathrm{~N} \mathrm{HCl}$ at $100^{\circ} \mathrm{C}$ for $24 \mathrm{hr}$. The 
hydrolysates were processed and analysed by reverse phase HPLC as described (Poinar et al., 1996). Amounts of the amino acid enantiomers were determined by comparison to standards after subtraction of the amino acid content determined in a negative control processed in parallel with the samples.

\section{DNA Extraction}

Bone pieces were removed using a drill saw. Subsequently, the surface of the pieces was removed with a grinding bit and furthermore treated by soaking in a $10 \%$ solution of $\mathrm{NaClO}$ for $10 \mathrm{~s}$, and rinsing in double-distilled and UV-irradiated $\mathrm{H}_{2} \mathrm{O}$. The samples were then ground to powder in a Spex Mill (Edison, $\mathrm{NJ}$ ) filled with liquid nitrogen. The powder was incubated in $1 \mathrm{ml}$ of $0.5 \mathrm{M}$ EDTA ( $\mathrm{pH} \mathrm{8.0),}$ $5 \%$ sarkosyl on a rotary wheel at ambient temperature for $\sim 40 \mathrm{hr}$. Ten microliters of proteinase $\mathrm{K}(10 \mathrm{mg} / \mathrm{ml})$ were then added and the incubation continued at $37^{\circ} \mathrm{C}$ for another $40 \mathrm{hr}$. Tissue remains were removed by centrifugation and the supernatant extracted with phenol, phenol/chloroform, and chloroform/isoamyl alcohol as described (Ausubel et al., 1995). The aquaeous phase was concentrated by centrifugal dialysis using Microcon-30 microconcentrators (Amicon, Beverly, MA) and incubated with $40 \mu$ l silica suspension in $1 \mathrm{ml}$ of $5 \mathrm{M}$ guanidinium isothiocyanate, $0.1 \mathrm{M}$ Tris- $\mathrm{HCl}(\mathrm{pH} 7.4)$ for $15 \mathrm{~min}$ on a rotary wheel at ambient temperature as described (Höss and Pääbo, 1993). The silica was collected by centrifugation and washed twice with $1 \mathrm{ml} 70 \%$ ethanol and once with $1 \mathrm{mlacetone}$, prior to air-drying. DNA was eluted into two aliquots of $65 \mu \mathrm{TE}(\mathrm{pH}$ 8.0) at $56^{\circ} \mathrm{C}$. The eluates were pooled, aliquoted, and stored at $-20^{\circ} \mathrm{C}$. An extraction control to which no bone powder was added was processed in parallel with each sample extraction.

\section{PCR, Cloning, and Sequencing}

A lower reaction mixture of $10 \mu \mathrm{l}(67 \mathrm{mM}$ Tris- $\mathrm{HCl}$ [pH 8.8], $2 \mathrm{mM}$ $\mathrm{MgCl}_{2}, 2 \mathrm{mg} / \mathrm{ml} \mathrm{BSA}, 2 \mu \mathrm{M}$ of each primer and $0.25 \mathrm{mM}$ of each dNTP) was separated prior to the first denaturation step by a wax layer from a $10 \mu$ l upper mixture $(67 \mathrm{mM}$ Tris- $\mathrm{HCl}$ [pH 8.8], $2 \mathrm{mM}$ $\mathrm{MgCl}_{2}, 0.375$ units Taq DNA polymerase [Perkin Elmer, Cetus], $5 \mu \mathrm{l}$ bone extract). Forty cycles of PCR $\left(15 \mathrm{~s}\right.$ at $92^{\circ} \mathrm{C}, 1 \mathrm{~min}$ at $55^{\circ} \mathrm{C}$ or $60^{\circ} \mathrm{C}, 1 \mathrm{~min}$ at $72^{\circ} \mathrm{C}$ ) were carried out in an MJ research PTC 200 cycler. Ten microliters of the reactions was electrophoresed in $3 \%$ agarose gels, stained with ethidium bromide, and visualized by UV transillumination. The bands, as well as the corresponding areas of the lanes where control amplifications were electrophoresed, were cut out of the gels, melted in $100 \mu \mathrm{l}$ double-distilled $\mathrm{H}_{2} \mathrm{O}$ and shock frozen in liquid nitrogen. After thawing during centrifugation, $5 \mu$ of the supernatant was used in $50 \mu \mathrm{l}$ amplification reactions $(67 \mathrm{mM}$ Tris- $\mathrm{HCl}[\mathrm{pH} 8.8$ ], $2 \mathrm{mM} \mathrm{MgCl}, 1 \mathrm{mg} / \mathrm{ml} \mathrm{BSA}, 1 \mu \mathrm{M}$ of each primer, $0.125 \mathrm{mM}$ of each dNTP, 0.75 units Taq DNA polymerase). Thirty cycles identical to the initial amplification, except for an increase of $3^{\circ} \mathrm{C}$ in annealing temperature, were performed. If primer dimers or nonspecific bands were visible upon gel electrophoresis, reamplification products were gel purified prior to cloning. Alternatively, 10 $\mu$ l of the reamplification volume were directly treated with T4 DNA polymerase (New England Biolabs) according to the supplier's protocol and ligated into a Smal-cut pUC18 (Pharmacia Biotech, Uppsala, Sweden) vector in the presence of 10 units of Smal at ambient temperature for $16 \mathrm{hr}$. E. coli SURE (Stratagene, La J olla, CA) were transformed by electroporation using half of the ligation and grown in $1 \mathrm{ml} \mathrm{SOC}$ medium (Ausubel et al., 1995) for 20-25 min before plating on selective IPTG/X-gal agar plates.

White colonies were transferred into $12.5 \mu \mathrm{l} P C R$ reactions (contents as in reamplifications) with "M 13 universal" and "M 13 reverse" primers. After $5 \mathrm{~min}$ at $92^{\circ} \mathrm{C}, 30$ cycles of PCR $\left(30 \mathrm{~s}\right.$ at $90^{\circ} \mathrm{C}, 1 \mathrm{~min}$ at $50^{\circ} \mathrm{C}, 1 \mathrm{~min}$ at $72^{\circ} \mathrm{C}$ ) were carried out and clones with inserts of the expected size were identified by agarose gel electrophoresis. Of these PCR products, $1.5 \mu \mathrm{l}$ was sequenced with the Thermo Sequenase kit (Amersham, UK) according to the supplier's instructions, and half of the sequencing reactions were loaded onto a $6.5 \%$ denaturing polyacrylamide gel and analysed on an A.L.F. automated sequencer (Pharmacia Biotech, Uppsala, Sweden).

\section{Quantitative PCR}

A competitor standard was constructed (Förster 1994; Handt et al. 1996) and cloned into pUC18. This molecule matches the Neandertal
mtDNA sequence from base 16,190 to 16,290 but lacks 12 bases $(16,210$ to 16,221$)$. The plasmid was purified from an overnight culture by alkaline lysis (Ausubel et al., 1995) and the DNA concentration determined by UV absorbance at $260 / 280 \mathrm{~nm}$. The standard and Neandertal DNA extracts were used in amplifications with two primers (NL16,209: 5'-CCC CAT GCT TAC AAG CAA GC-3', NH16,262: 5'-GTA GAT TTG TTG ATA TCC TAG TGG GTG TAA-3') specific for the Neandertal sequence.

\section{Sequence Analyses}

In total, 27 amplifications of the mtDNA control region were performed from the Neandertal specimen. Of these, twelve amplifications yielded exclusively Neandertal sequences, whereas 6 amplifications contained sequences similar to the contemporary human reference sequence as well as Neandertal sequences. Figures 2, 4, and 5 give all Neandertal sequences determined except those from the quantitation reaction (Figure 3 ). In addition, 9 amplifications yielded exclusively sequences similar to the human reference sequence (not shown). In these cases, the primers used turned out to have one to four mismatches to the Neandertal sequence and thus to select against the latter. The Neandertal sequence was inferred using the sequences shown in Figures 2, 4, and 5.

The Neandertal sequence was compared to an mtDNA sequence database (S. Meyer, personal communication). Except for the scoring of variable positions, only human sequences where no insertions, deletions, or ambiguities occur were used. In the case of the 16 chimpanzee lineages, four are reported (Morin et al., 1994) with an ambiguity at position 16,049, and one each with ambiguities at positions 16,063 and 16,064 . Pairwise sequence differences were determined using unpublished software by $A$. von Haeseler. Maximum likelihood distances and phylogenetic trees were computed using the PHYLIP package, version 3.5 (Felsenstein, 1994), assuming a transition/transversion ratio of 20. Support for internal branches was tested by likelihood mapping using the program Puzzle 3.0 (Strimmer and von Haeseler, 1997). Briefly, this algorithm analyzes all possible quartets of sequences, two from one side of an internal branch, two from the other. In each case, enough subsamples of sequences were analyzed to ensure with $95 \%$ probability that any particular sequence was sampled at least once.

For dating, the Tamura-Nei algorithm (Tamura and Nei, 1993) as implemented in Puzzle 3.0 was used to estimate 9 classes of substitution rates, rate heterogeneity parameters, transition/transversion ratios, pyrimidine/purine transition ratios and nucleotide frequencies for 5 datasets consisting of 100 random human sequences. The average values for these parameters were then used to calculate the distances within and between species.

\section{Acknowledgments}

We are indebted to Drs. F. G. Zehnder and H.-E. J oachim (Rheinisches Landesmuseum Bonn) for permission to remove the sample; to $H$. Lüdtke and M. Schultz for support and advice in the sampling process; to S. Meyer and K. Strimmer for help with computer analyses; to W. Schartau for oligonucleotide synthesis; to M. Beutelspacher, H. Fröhlich, A. Greenwood, R. F. Grill, M. Höss, T. Merritt, $H$. Poinar, L. Vigilant, and $H$. Zischler for discussions and help; to the Deutsche Forschungsgemeinschaft ( $\mathrm{Pa}$ 452/3-1), the Boehringer Ingelheim Fonds (M. K.), and the National Science Foundation (M. S.) for financial support. R. W. S. especially thanks his late Ph.D. supervisor W. Taute (University of Cologne).

Received April 24, 1997; revised J une 16, 1997.

\section{References}

Anderson, S., Bankier, A.T., Barrell, B.G., de Bruijn, M.H.L., Coulson, A.R., Drouin, J., Eperon, I.C., Nierlich, D.P., Roe, B.A., Sanger, F., et al. (1981). Sequence and organization of the human mitochondrial genome. Nature 290, 457-474.

Armour, J .A.L., Anttinen, T., May, C.A., Vega, E.E., Sajantila, A., Kidd, J .R., Kidd, K.K., Bertranpetit, J ., Pääbo, S., and J effreys, A.J . (1996). Minisatellite diversity supports a recent African origin for modern humans. Nature Genet. 13, 154-160. 
Ausubel, F.A., Brent, R., Kingston, R.E., Moore, D.D., Seidman, J .G., Smith, J .A., and Struhl, K., eds. (1995). Current Protocols in Molecular Biology (New York: J ohn Wiley \& Sons).

Bendall, K.E., and Sykes, B.C. (1995). Length heteroplasmy in the first hypervariable segment of the human mtDNA control region. Am. J. Hum. Genet. 57, 248-256.

Bräuer, G., and Stringer, C.B. (1997). Models, polarisation, and perspectives on modern human origins. In Conceptual Issues in Modern Human Origins Research, G.A. Clark and C.M. Willermet, eds. (New York: de Gruyer), in press.

Cann, R.L., Stoneking, M., and Wilson, A.C. (1987). Mitochondrial DNA and human evolution. Nature 325, 31-36.

Collura, R.V., and Stewart, C.-B. (1995). Insertions and duplications of mtDNA in the nuclear genomes of Old World monkeys and hominoids. Nature $378,485-489$

Comas, D., Pääbo, S., and Bertranpetit, J . (1995). Heteroplasmy in the control region of human mitochondrial DNA. Genome Res. 5 , 89-90.

Felsenstein, J. (1994). Phylip 3.5 (University of Washington, Seattle).

Foley, R., and Lahr, M.M. (1997). Mode 3 technologies and the evolution of modern humans. Cambridge Archaeol. J ., 7, 3-36.

Förster, E. (1994). An improved general method to generate internal standards for competitive PCR. Biotechniques 16, 18-20.

Hammer, M.F. (1995). A recent common ancestry for human $Y$ chromosomes. Nature 378, 376-378.

Handt, O., Höss, M., Krings, M., and Pääbo, S. (1994a). Ancient DNA: methodological challenges. Experientia 50, 524-529.

Handt, O., Richards, M., Trommsdorff, M., Kilger, C., Simanainen, J ., Georgiev, O., Bauer, K., Stone, A., Hedges, R., Schaffner, W. Utermann, G., Sykes, B., and Pääbo, S. (1994b). Molecular genetic analyses of the Tyrolean ice man. Science 264, 1775-1778.

Handt, O., Krings, M., Ward, R.H., and Pääbo, S. (1996). The retrieval of ancient human DNA sequences. Am. J. Hum. Genet. 59, 368-376. Harpending, H.C., Sherry, S.T., Rogers, A.R., and Stoneking, M. (1993). The genetic structure of ancient human populations. Curr. Anthropol. 34, 483-496.

Hedges, B., Kumar, S., Tamura, K., and Stoneking, M. (1992). Human origins and analysis of mitochondrial DNA sequences. Science 255 737-739.

Höss, M., and Pääbo, S. (1993). DNA extraction from Pleistocene bones by a silica-based purification method. Nucleic Acids Res. 21 , 3913-3914.

Höss, M., Jaruga, P., Zastawny, T.H., Dizdaroglu, M., and Pääbo, S. (1996a). DNA damage and DNA sequence retrieval from ancient tissues. Nucleic Acids Res. 24, 1304-1307.

Höss, M., Dilling, A., Currant, A., and Pääbo, S. (1996b). Molecular phylogeny of the extinct ground sloth Mylodon darwinii. Proc. Natl. Acad. Sci. USA 93, 181-185.

Hublin, J. . . Spoor, F., Braun, M., Zonneveld, F., and Condemi, S. (1996). A late Neanderthal associated with Upper Paleolithic artefacts. Nature 381, 224-226.

Ivanov, P.L., Wadhams, M.J., Roby, R.K., Holland, M.M., Weedn, V.W., and Parsons, T.J. (1996). Mitochondrial DNA sequence heteroplasmy in the Grand Duke of Russia Georgij Romanov establishes the authenticity of the remains of Tsar Nicholas II. Nature Genet. $12,417-420$

Lindahl, T. (1993a). Instability and decay of the primary structure of DNA. Nature 362, 709-715

Lindahl, T. (1993b). Recovery of antediluvian DNA. Nature 365, 700 Maddison, D.R. (1991). African origin of human mitochondrial DNA reexamined. Syst. Zool. 40, 355-363.

Morin, P.A., Moore, J .J ., Chakraborty, R., J in, L., Goodall, J., and Woodruff, D.S. (1994). Kin selection, social structure, gene flow, and the evolution of chimpanzees. Science 265, 1193-1201.

Mullis, K.B., and Faloona, F. (1987). Specific synthesis of DNA in vitro via a polymerase-catalyzed chain reaction. Methods Enzymol. $155,335-350$

Nei, M. (1987). Molecular Evolutionary Genetics (New York: Columbia University Press).
Pääbo, S. (1989). Ancient DNA: extraction, characterization, molecular cloning and enzymatic amplification. Proc. Natl. Acad. Sci. USA 86, 1939-1943.

Pääbo, S., and Wilson, A.C. (1991). Miocene DNA sequences-a dream come true? Current Biol. 1, 45-46.

Pääbo, S., Higuchi, R.G., and Wilson, A.C. (1989). Ancient DNA and the polymerase chain reaction. The emerging field of molecular archaeology. J . Biol. Chem. 264, 9709-9712.

Pääbo, S., Irwin, D.M., and Wilson, A.C. (1990). DNA damage promotes jumping between templates during enzymatic amplification. J. Biol. Chem. 265, 4718-4721.

Penny, D., Steel, M., Waddell, P.J., and Hendy, M.D. (1995). Improved analyses of human mtDNA sequences support a recent African origin for Homo sapiens. Mol. Biol. Evol. 12, 863-882.

Petri, B., von Haeseler, A., and Pääbo, S. (1996). Extreme sequence heteroplasmy in bat mitochondrial DNA. Biol. Chem. Hoppe-Seyler $377,661-667$

Poinar, H.N., Höss, M., Bada, J .L., and Pääbo, S. (1996). Amino acid racemization and the preservation of ancient DNA. Science 272 , 864-866.

Rak, Y. (1993). Morphological variation in Homo neanderthalensis and Homo sapiens in the Levant. A biogeographic modern. In Species, Species Concepts and Primate Evolution, W.H. Kimbel and L.B. Martin, eds. (New York: Plenum Press), 523-536.

Saitou, N., and Nei, M. (1987). The neighbor joining method: a new method for reconstructing phylogenetic trees. Mol. Biol. Evol. 4, 406-425.

Schmitz, R.W. (1996). Das Alt- und Mittelpaläolithikum des Neandertals und benachbarter Gebiete. Doctoral dissertation, Prehistory, University of Cologne (Cologne, University Microfilms)

Schmitz, R.W., Pieper, P., Bonte, W., and Krainitzki, H. (1995). New investigations of the Homo sapiens neanderthalensis, found in 1856. In Advances in Forensic Sciences, Vol. 7: Forensic Odontology and Anthropology. Proceedings of the 13th Meeting of the International Association of Forensic Sciences, Düsseldorf, August 22nd to 28th, 1993, B. J acob and W. Bonte, eds. (Berlin, Köster), pp. 42-44.

Schwartz, J., and Tattersall, I. (1996). Significance of some previously unrecognized apomorphies in the nasal region of Homo neanderthalensis. Proc. Natl. Acad. Sci. USA 93, 10852-10854.

Strimmer, K.S., and von Haeseler, A. (1997). Likelihood mapping: a simple method to visualize phylogenetic content of a sequence alignment. Proc. Natl. Acad. Sci. USA, 94, 6815-6819.

Stringer, C. (1997). Chronological and biogeographic perspectives on later human evolution. In Neanderthals and Modern Humans in West Asia, O. Bar-Yosef and T. Akazawa, eds. (New York: Plenum Press), in press

Stringer, C., and Gamble, C. (1993). In Search of the Neanderthals (London: Thames and Hudson).

Takahata, N., Satta, Y., and Klein, J . (1995). A genetic perspective on the origin and history of humans. Annu. Rev. Ecol. Syst. 26, 343-372.

Tamura, K., and Nei, M. (1993). Estimation of the number of nucleotide substitutions in the control region of mitochondrial DNA in humans and chimpanzees. J. Mol. Evol. 10, 512-526.

Templeton, A.R. (1992). Human origins and analysis of mitochondrial DNA sequences. Science 255, 737.

Tishkoff, S.A., Dietsch, E., Speed, W., Pakstis, A.J., Kidd, J.R., Cheung, K., Bonné-Tamir, B., Santachiara-Benerechetti, A.S., Moral, P., Krings, M., et al. (1996). Global patterns of linkage disequilibrium at the CD4 locus and modern human origins. Science 271, 13801387.

Trinkaus, E., and Shipman, P. (1993). The Neandertals: Changing the Image of Mankind (New York: Knopf).

van der Kuyl, A., Kuiken, C.L., Dekker, J .T., Perizonius, W.R.K., and Goudsmit, J. (1995). Nuclear counterparts of the cytoplasmic mitochondrial 12s rRNA gene: a problem of ancient DNA and molecular phylogenies. J. Mol. Evol. 40,652-657.

Vigilant, L., Stoneking, M., Harpending, H., Hawkes, K., and Wilson, A.C. (1991). African populations and the evolution of mitochondrial DNA. Science 253, 1503-1507. 
von Haeseler, A., Sajantila, A., and Pääbo, S. (1996). The genetical archaeology of the human genome. Nature Genet. 14, 135-140.

Wolpoff, M.H. (1989). Multiregional evolution: the fossil alternative to Eden. In The Human Revolution, C. Stringer and P. Mellars, eds. (Edinburgh: Edinburgh University Press).

Zischler, H., Geisert, H., von Haeseler, A., and Pääbo, S. (1995). A nuclear 'fossil' of the mitochondrial D-loop and the origin of modern humans. Nature $378,489-492$.

Zollikofer, C., Ponce de Leon, M., Martin, R., and Stucki, P. (1995). Neanderthal computer skulls. Nature 375, 283-285.

\section{GenBank Accession Number}

The GenBank accession number for the Neandertal sequence reported here is AF011222. 